\title{
Aspectos clínicos e radiográficos de pacientes com síndrome do impacto femoroacetabular: Há diferença entre quadril sintomático e assintomático?*
}

\section{Clinical and Radiographic Aspects of Patients with Femoroacetabular Impingement Syndrome: Is There Difference between Symptomatic and Asymptomatic Hip?}

\author{
Andreza Maroneze Silva ${ }^{1}$ Flávia Marques Nakatake ${ }^{1}$ Maria Cristina Arruda Neves ${ }^{1}$ \\ Vera Lúcia Santos Alves ${ }^{1}$ Giancarlo Cavalli Polesello ${ }^{1}$ \\ ${ }^{1}$ Irmandade da Santa Casa de Misericórdia de São Paulo, Faculdade de \\ Ciências Médicas, São Paulo, SP, Brasil \\ Endereço para correspondência Vera Lúcia dos Santos Alves, PhD, \\ Irmandade da Santa Casa de Misericórdia de São Paulo, Faculdade de \\ Ciências Médicas, Rua Dr. Cesário Mota Júnior, 112, Vila Buarque - \\ São Paulo, SP 01221-020, Brasil \\ Rev Bras Ortop 2020;55(2):247-253. \\ (e-mail: fisioterapiasc@uol.com.br; andreza.fisio.orto@gmail.com).
}

\begin{abstract}
Resumo
Objetivos Comparar características clínicas e radiográficas entre quadril sintomático e assintomático em pacientes com síndrome do impacto femoroacetabular unilateral e estabelecer correlações entre os achados.

Métodos Estudo retrospectivo, que consultou prontuários de pacientes com síndrome do impacto femoroacetabular, entre janeiro de 2014 e abril de 2017. Os pacientes foram avaliados clinicamente pelo questionário International Hip Outcome Tool 33 (iHOT33), escala visual analógica de dor, amplitude de rotação de quadril e força muscular de quadril e joelho. A avaliação radiográfica foi composta por mensurações do ângulo alfa, sinal do cruzamento, índice de retroversão acetabular, sinal da espinha isquiática e sinal da parede posterior do acetábulo.

Resultados Foram incluídos no estudo 45 prontuários de pacientes, com tempo médio de sintomas até o diagnóstico de 28,6 meses e pontuação média no iHOT33 de

Palavras-chave

- impacto femoroacetabular

- quadril

- amplitude de movimento 39,9. O valor médio de rotação medial do quadril sintomático foi de $20,5^{\circ}$ e do assintomático $27,2^{\circ}$, com $(p<0,001)$. A positividade do sinal do cruzamento para quadril sintomático foi de $68,9 \%$ e do assintomático $55,6 \%(p=0,03)$. Para índice de retroversão, o valor médio do quadril sintomático foi de 0,15 e do quadril assintomático foi $0,11(p=0,02)$. Encontramos correlação positiva entre o tempo de sintomas e a redução de amplitude de rotação medial de quadril $(p=0,04)$ e entre o índice de massa corpórea (IMC) e a redução amplitude de rotação medial de quadril $(p=0,02)$.
\end{abstract}

* Trabalho desenvolvido no Departamento de Fisioterapia Músculo-Esquelética da Irmandade da Santa Casa de Misericórdia de São Paulo, São Paulo, SP, Brasil. recebido

05 de Maio de 2018

aceito

22 de Janeiro de 2019
DOI https://doi.org/

10.1055/s-0039-3400519. ISSN 0102-3616.
Copyright $\odot 2020$ by Sociedade Brasileira License terms de Ortopedia e Traumatologia. Published by Thieme Revinter Publicações Ltda, Rio de Janeiro, Brazil 


\begin{abstract}
Keywords

- femoroacetabular impingement

- hip

- range of motion

Objective This paper aims to compare clinical and radiographic features of symptomatic and asymptomatic hip in patients with unilateral femoroacetabular impingement syndrome (FAIS) and to establish correlation between these findings.

Methods This is a retrospective study, based on medical records of patients diagnosed with FAIS between January 2014 and April 2017. The patients were assessed clinically as per the International Hip Outcome Tool 33 (iHOT33) questionnaire, visual analogue pain scale, hip rotation, and hip and knee muscular strength. The radiographic evaluation consisted of measurements of the alpha angle, crossover signal, acetabular retroversion index, ischial spine signal, and posterior wall sign.

Results A total of 45 patients were included in the study, with mean time from symptom onset to diagnosis of 28,6 months and mean iHOT33 score of 39.9. The mean medial rotation was $20.5^{\circ}$ in the symptomatic hip and $27.2^{\circ}$ in the asymptomatic hip $(p<0.001)$. The crossover signal was positive on $68.9 \%$ of the symptomatic hips and $55,6 \%$ of the asymptomatic hips $(p=0.03)$. The mean retroversion index was 0.15 in symptomatic hips and 0.11 in asymptomatic hips $(p=0.02)$. There was a positive correlation between the total time of symptoms and reduction of medial hip rotation $(p=0.04)$ and between body mass index (BMI) and reduction in medial hip rotation $(p=0.02)$.

Conclusion When comparing clinical and radiographic characteristics, we observed reduction of medial rotation and increase of acetabular retroversion index in the symptomatic hip, as well as association between the long symptom time and the high BMI with loss of medial rotation of the hips.
\end{abstract}

Conclusão Ao comparar características clínicas e radiográficas, observamos redução de rotação medial e aumento do índice de retroversão acetabular no quadril sintomático, bem como associação entre o longo tempo de sintoma e o IMC elevado com perda de rotação medial dos quadris.

\section{Introdução}

A síndrome do impacto femoroacetabular (SIFA) é definida como distúrbio clínico relacionado ao movimento do quadril, composto por uma tríade - sintomas, sinais clínicos, e achados de imagem. Divide-se em três subtipos clínicos: came (alteração morfológica femoral), pincer (alteração morfológica acetabular), e misto (combinação). ${ }^{1}$

Na fisiopatologia da síndrome, existe o contato anormal entre a extremidade proximal do fêmur e acetábulo durante os extremos de amplitude de movimento de quadril e, de forma repetida, pode gerar lesões progressivas no lábio e na cartilagem acetabular. A condição é conhecida como causa comum de dor no quadril e ganhou reconhecimento por ter correlação com o desenvolvimento da osteoartrite. ${ }^{2}$

O principal sintoma da síndrome é a dor anterior/inguinal do quadril e a sobrecarga mecânica decorrente do impacto pode provocar alterações funcionais em todo membro inferior, tais como redução de amplitude de movimento (ADM), mudanças na produção de força, controle neuromuscular, marcha e tarefas esportivas mais intensas. Uma revisão sistemática conclui que esta população realiza compensações nos planos frontal, sagital, e transversal durante atividades dinâmicas comparadas a controles assintomáticos. ${ }^{3}$
Na literatura são relatados trabalhos que comparam parâmetros clínicos e radiográficos de pacientes com SIFA em relação a controles assintomáticos, bem como estudos que confrontam características do membro dominante em relação ao não dominante. ${ }^{4}$ Há escassez, porém, de estudos que comparem o membro sintomático em relação ao assintomático em pacientes com SIFA unilateral, de forma a ampliar a compreensão das características que levam o quadril a se tornar doloroso.

O objetivo deste estudo foi comparar características clínicas e radiográficas entre quadril sintomático e assintomático em pacientes com SIFA unilateral e estabelecer uma correlação entre os achados.

\section{Método}

Tratou-se de estudo retrospectivo, observacional, clínico, auto-controlado, desenvolvido com a consulta de prontuários em um centro único. Os prontuários consultados foram de pacientes acompanhados de janeiro de 2014 a abril de 2017 de casuística de único cirurgião (Giancarlo Cavalli Polesello - GCP). O estudo foi aprovado pelo Comitê de Ética em Pesquisa com CAAE número 63881917.8.0000.5479 e escrita do artigo foi guiada pelo checklist Strengthening the Reporting of Observational studies in Epidemiology (Strobe). ${ }^{5}$ 
Os critérios de inclusão foram: prontuários completos com informação de pacientes de ambos os sexos com diagnóstico de SIFA unilateral, idade entre 18 e 55 anos e assinatura do termo de consentimento livre e esclarecido. Foram excluídos prontuários de pacientes com cirurgia ortopédica prévia de coluna vertebral e/ou membros inferiores, osteoartrite degenerativa do quadril, sinovite vilonodular, condromatose sinovial, tumores malignos do quadril e relato de uso contínuo de analgésicos opióides no momento de realização dos testes funcionais.

Foram examinados os prontuários de 95 pacientes com a casuística final sendo constituída por 45 pacientes. Foram examinados os dados de ambos os quadris, o que constituiu uma amostra de 90 quadris.

O diagnóstico da SIFA foi confirmado pela anamnese, exame físico e exames de imagem. Os testes clínicos aplicados foram o flexão, adução e rotação interna (FADIR, na sigla em inglês) e o FABERE (flexão, abdução e rotação externa (FABER, na sigla em inglês ${ }^{6}$ e os exames de imagem foram os de radiografia anteroposterior (AP) de pelve em ortostatismo, perfil do colo femoral (Dunn ou Ducroquet) e ressonância magnética nos pacientes com indicação cirúrgica, para melhor avaliação de lábio e cartilagem acetabular. Na dúvida diagnóstica após toda rotina foi feita injeção intrarticular de anestésico e reavaliação de sinais e sintomas. Todos os pacientes foram avaliados por um cirurgião sênior de quadril ( $>20$ anos de experiência) e por equipe de reabilitação treinada na área de quadril há mais de 5 anos.

\section{Avaliação Clínica e Funcional}

Em uma ficha padronizada foram extraídos dados para a caracterização clínica da amostra, tais como: sexo, idade (anos), peso (kg), altura ( $\mathrm{cm}$ ), índice de massa corpórea (IMC, $\mathrm{kg} / \mathrm{m}^{2}$ ), membro dominante, membro sintomático, tempo de sintomas (meses), doenças associadas, medicamentos em uso e nível de atividade física ( - Tabela $\mathbf{1}$ ).

Também houve a coleta de dados clínicos tais como, a pontuação da escala visual analógica (EVA) de dor, ADM de rotação medial (RM) e rotação lateral (RL) de quadril, valores de força muscular de quadril e joelho, questionário de qualidade de vida International Hip Outcome Tool 33 (iHOT33) e mensurações de radiografia AP de pelve (ângulo alfa, sinal do cruzamento, índice de retroversão acetabular, sinal da parede posterior e sinal da espinha isquiática). As avaliações foram realizadas por duas fisioterapeutas com 5 anos de experiência em reabilitação de quadril.

As mensurações das amplitudes de rotação do quadril foram realizadas com auxílio de um goniômetro universal (Carci, São Paulo, SP, Brasil) com o paciente em decúbito dorsal e pelve estabilizada na maca com auxílio de cinto para evitar compensações. $\mathrm{O}$ membro avaliado foi posicionado em flexão de quadril a $90^{\circ}$ e o membro contralateral em extensão. 0 eixo do goniômetro foi posicionado sobre o centro do joelho do lado a ser medido, sendo o braço fixo paralelo ao solo e o móvel seguiu o eixo da tíbia, realizando a rotação medial e lateral do fêmur dos participantes (-Figura 1).? Os avaliadores possuíam índice de correlação de classes (ICC) acima de 0,80 para avaliação da ADM de rotação de quadril.
Tabela 1 Descrição das variáveis antropometricas e analíticas dos 45 pacientes que compuseram a amostra do estudo

\begin{tabular}{|c|c|c|}
\hline Sexo & & \\
\hline Feminino & $28(62.2 \%)$ & \\
\hline Masculino & $17(37.8 \%)$ & \\
\hline Idade (anos) & $39.02 \pm 8.03$ & \\
\hline Peso $(\mathrm{kg})$ & $69.63 \pm 14.52$ & \\
\hline Altura $(\mathrm{cm})$ & $168.89 \pm 9.86$ & \\
\hline IMC $\left(\mathrm{kg} / \mathrm{m}^{2}\right)$ & $24.16 \pm 3.21$ & \\
\hline $\begin{array}{l}\text { Duração dos } \\
\text { sintomas (meses) }\end{array}$ & $28.64 \pm 31.91$ & \\
\hline EVA $(0-10)$ & $5.67 \pm 2.55$ & \\
\hline iHOT-33 (0-100) & $39.93 \pm 21.20$ & \\
\hline \multicolumn{3}{|l|}{ Local da dor } \\
\hline Sinal do C & 22 (48.9\%) & \\
\hline Inguinal & $17(37.8 \%)$ & \\
\hline Trocânter & $6(13.3 \%)$ & \\
\hline \multicolumn{3}{|l|}{ Membro dominante } \\
\hline Direito & $43(95.6 \%)$ & \\
\hline Esquerdo & $2(4.4 \%)$ & \\
\hline \multicolumn{3}{|c|}{ Membro sintomático } \\
\hline Direito & $29(64.4 \%)$ & \\
\hline Esquerdo & $16(35.6 \%)$ & \\
\hline Subtipo de IFA & Sintomático & Assintomático \\
\hline Cam & $13(28.9 \%)$ & $15(33.3 \%)$ \\
\hline Pincer & $12(26.6 \%)$ & $09(20 \%)$ \\
\hline Misto & 20 (44.5\%) & $18(40 \%)$ \\
\hline \multicolumn{3}{|l|}{ Atividade física } \\
\hline Sedentário & $16(35.6 \%)$ & \\
\hline Ativo & $29(64.4 \%)$ & \\
\hline
\end{tabular}

Abbreviations: DP,desvio padrão; EVA, escala visual analógica; IFA, impacto femoroacetabular; iHOT-33, The international HipOutcome Tool-33; IMC, índice de massa corpórea.

Para mensurar a força muscular de quadril e joelho foi utilizado dinamômetro manual MicroFet2 (Hoggan Health Industries Inc., West Jordan, UT, EUA). ${ }^{8}$ Para evitar compensações, o paciente foi posicionado com cinto de estabilização (-Figura 2). ${ }^{9}$ Foram realizadas três contrações isométricas de cinco segundos cada, a média das coletas foi selecionada e os grupos musculares avaliados foram flexores, extensores, adutores, abdutores, rotadores mediais e laterais de quadril, flexores e extensores de joelho. ${ }^{10} \mathrm{~A}$ força foi medida em quilogramas $(\mathrm{kg})$ e normalizada para massa corporal $(\mathrm{kg})$, com a fórmula: (força $[\mathrm{kg}] /$ peso corporal $[\mathrm{kg}]$ ) x $100 .{ }^{11} \mathrm{Os}$ avaliadores possuíam ICC acima de 0,80 para avaliação da mensuração de força com dinamômetro manual dos grupos musculares de quadril e joelho.

Para avaliar limitações da SIFA na qualidade de vida dos indivíduos, foi utilizada a pontuação do iHOT33. ${ }^{12}$ O questionário é utilizado em jovens adultos com quadril não 


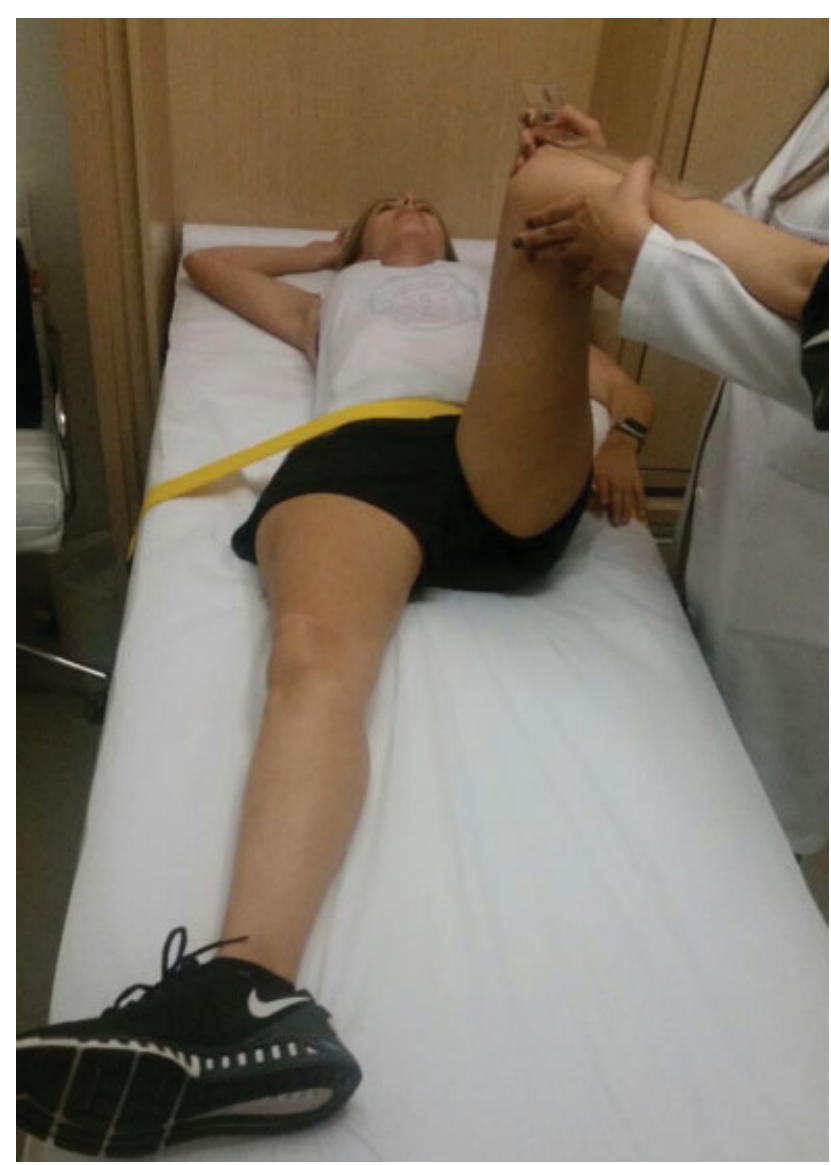

Fig. 1 Posicionamento do paciente e mensuração da amplitude de rotação medial do quadril com estabilização.

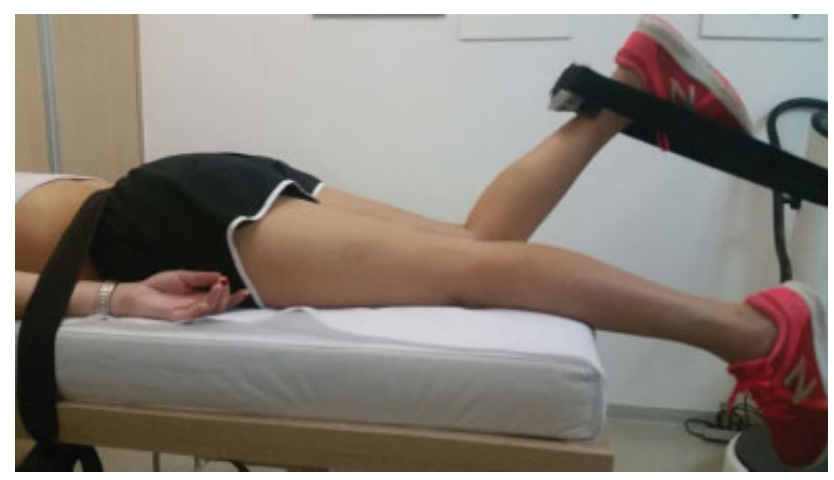

Fig. 2 Imagem ilustrativa para medida da força muscular de flexores de joelho com dinamômetro isométrico manual e estabilização com cinto.

artrítico, traduzido e adaptado para língua portuguesa em $2012 .{ }^{13}$ Foi de autopreenchimento, com 33 questões divididas em quatro domínios: sintomas e limitações funcionais, esportes e atividades recreativas, considerações relacionadas ao trabalho e considerações sociais, emocionais e sobre estilo de vida. Estas questões são avaliadas por escala com linha que possui $100 \mathrm{~mm}$ de comprimento. A questão foi respondida com o paciente orientado a traçar uma barra na linha. 0 escore final foi a somatória das questões respondidas, dividido pelo número total de questões respondidas.

\section{Avaliação Radiográfica}

Todos pacientes realizaram radiografia digital AP da bacia em ortostatismo, com rotação interna de $15^{\circ}$ dos membros inferiores, com raio centrado $1 \mathrm{~cm}$ acima da sínfise púbica, a ampola a $120 \mathrm{~cm}$ do chassi e aumento de $100 \%$ para avaliar ângulo alfa, índice de retroversão acetabular, sinais da espinha isquiática, do cruzamento e da parede posterior. ${ }^{14}$

$\mathrm{O}$ ângulo alfa é a expressão quantitativa da deformidade femoral. Foi avaliado segundo método descrito por Gosvig et al. ${ }^{15} \mathrm{e}$ os pontos de referência para traçar o ângulo foram centro do colo femoral, centro da cabeça femoral e início da formação da deformidade femoral ( - Figura 3A). Os autores categorizaram valores para o ângulo alfa na radiografia de bacia AP em ortostase, em normal, limítrofe e patológico. Definiu-se como critério diagnóstico de morfologia came no presente estudo, valores de ângulo alfa patológicos ( $\geq 83^{\circ}$ para homens e $\geq 57^{\circ}$ para mulheres).

$O$ índice de retroversão acetabular (IRA) expressa de forma quantitativa o sinal do cruzamento, que representa sinal radiográfico de hipercobertura acetabular anterior e foi medido segundo Diaz-Ledezma et al. ${ }^{16}$ em 2013. A mensuração do IRA foi o quociente entre comprimento de sobreposição da borda anterior em comparação com a distância lateral da cavidade acetabular. Valores acima de 0,20 têm relação com lesão condral ( - Figura 3B). O sinal da espinha isquiática, sinal da parede posterior e sinal do cruzamento foram descritos como presentes ou ausentes.

Foi utilizado o software HOROS, visualizador de imagem (64-bit) gratuito, para OS X. O software baseia-se no OsiriX e em outras bibliotecas de imagens médicas de código aberto, sendo disponibilizado sob a GNU General Public License, versão 3 (GPL-3.0).

A avaliação das imagens foi realizada por médico ortopedista com atuação na área de cirurgia do quadril há mais de cinco anos, de forma cega quanto ao nome do paciente e membro sintomático e foi realizado ICC em estudo anterior, com boa correlação em todos os parâmetros analisados. ${ }^{17}$

\section{Análise Estatística}

Foram utilizados programas Excel Office 2017 (Microsoft Corp., Redmond, WA, EUA) e o SPSS 13.0 (SPSS Inc., Chicago, IL, EUA). Os dados descritivos foram expressos em média e desvio padrão. A comparação entre variáveis foi feita pelos testes de Wilcoxon, $\mathrm{T}$ de Student pareado, Mcnemar, e Pearson. $\mathrm{O}$ valor de $p$ adotado como significante foi de 0,05 .

\section{Resultados}

O valor médio da amplitude de rotação medial no quadril sintomático foi de $20,56^{\circ} \pm 12,39^{\circ}$ e no quadril assintomático de $27,22^{\circ} \pm 12,59^{\circ}(p<0,001)$. Quanto à rotação lateral os valores foram respectivamente, $40,89^{\circ} \pm 8,2^{\circ}$ e $44,67^{\circ} \pm 5,7^{\circ}$ $(p=0,003)$.

$\mathrm{Na}$ análise de força muscular, ao comparar médias entre os grupos musculares do quadril e joelho, membro sintomático e assintomático, encontramos diferença significante nos adutores do quadril $(p=0,040)$ e flexores do joelho $(p=0,007)$. Os demais grupos não apresentaram diferença 

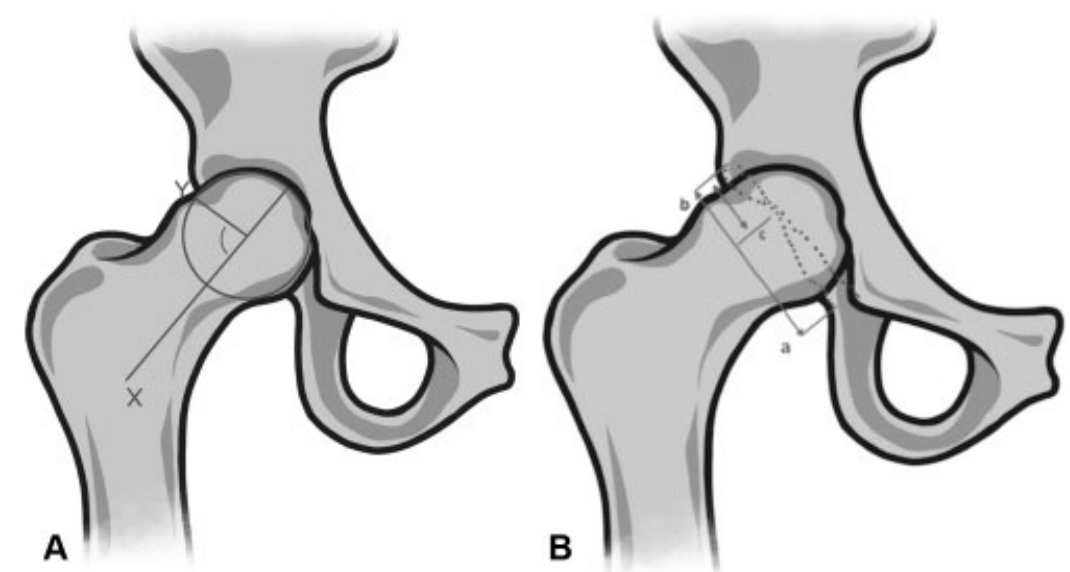

Fig. 3 Traçados para mensurações das medidas radiográficas. (A) ângulo alfa; (B) índice de retroversão.

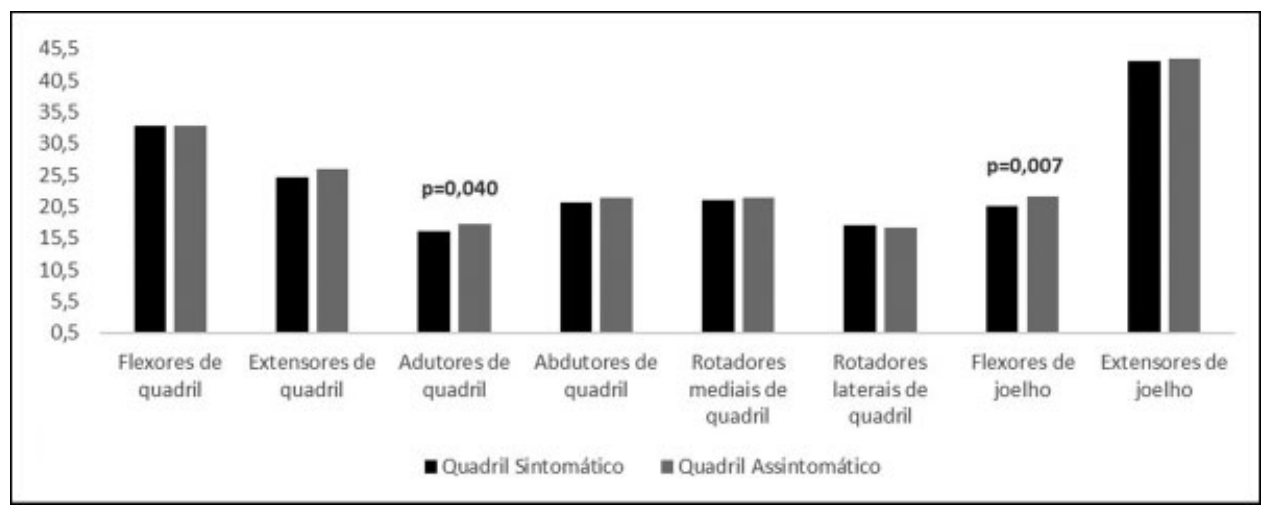

Fig. 4 Gráfico que representa valores de dinamometria de quadril e joelho normalizada para peso corporal - comparação entre quadril sintomático e assintomático ( $\mathrm{n}=90$ quadris).

com: flexores de quadril $(p=0,915)$, extensores de quadril $(p=0,082)$, abdutores de quadril $(p=0,090)$, rotadores mediais de quadril $(p=0,378)$, rotadores laterais de quadril $(p=0,345)$ e extensores de joelho $(p=0,942)$ ( - Figura 4$)$.

Quanto à análise radiográfica na comparação entre membros, o valor médio do ângulo alfa no membro sintomático foi de $74,06^{\circ} \pm 7,57$ e no assintomático $71,76^{\circ} \pm 7,07(p=0,080)$. Para o índice de retroversão, os valores foram de 0,15 $\pm 0,11$ e $0,11 \pm 0,11$, respectivamente $(p=0,009)$. A positividade do sinal do cruzamento para membro sintomático foi de $68,9 \%$ (31 quadris) e para assintomático foi de 55,6\% (25 quadris) $(p=0,030)$. Para sinal da espinha a positividade foi de $37,8 \%$ (17 quadris) para ambos membros $(p=1,000)$. Já o sinal da parede posterior teve positividade de $42,2 \%$ (19 quadris) no membro sintomático e $40 \%$ (18 quadris) para o assintomático $(p=1,000)$.

Na correlação entre todos os parâmetros avaliados houve significância estatística entre tempo de sintomas e perda de amplitude de rotação medial do quadril sintomático $(p=0,040) ; r=0,279$ e entre IMC e perda de amplitude de rotação medial no quadril sintomático $(p=0,020) ; r=0,495$.

\section{Discussão}

Os principais achados, ao comparar quadril sintomático e assintomático foram redução de amplitude de movimento de rotação medial e lateral de quadril, redução de força muscular dos grupos adutores de quadril e flexores de joelho, positividade do sinal do cruzamento e aumento do índice de retroversão acetabular no quadril sintomático. Ao estabelecer correlação entre tópicos avaliados, observamos que o tempo de sintomas apresentou fraca correlação com a redução de RM dos quadris e IMC apresentou moderada correlação a redução de RM dos quadris.

A mensuração da ADM do quadril é elemento importante da avaliação funcional. Há forte evidência de que a perda de amplitude de rotação medial do quadril é fator de risco para desenvolvimento de lesão intra-articular. Revisão sistemática ${ }^{18}$ indica que diferença acima de $7^{\circ}$ entre membros em relação à rotação medial ou lateral de quadril pode ser marcador para risco de lesão futura. Audenaert et al. ${ }^{19}$ encontraram redução significativa de amplitude de RM ao comparar grupo controle com indivíduos com SIFA e morfologia came e pincer. Esta alteração implica na qualidade de vida e prática esportiva, principalmente nas atividades com incremento de flexão de quadril.

Em 2016, foi estabelecido o acordo de Warwick, que orienta solicitação de radiografia simples AP da bacia e perfil do colo femoral para investigar alterações morfológicas e excluir outras causas de dor no quadril. ${ }^{1}$ Neste trabalho utilizamos a radiografia AP da bacia com carga, onde foi possível avaliar esfericidade femoral e sinais de retroversão 
acetabular. Sabemos que marcadores que avaliam sinais de pincer não são bem estabelecidos na literatura, por isso além dos três sinais mais utilizados, ${ }^{20}$ incluímos o índice de retroversão acetabular, que fornece informações quantitativas sobre a gravidade da hipercobertura focal e pode ser preditor de prognóstico em pacientes sintomáticos. ${ }^{16}$

Uma revisão sistemática de 2015 que avaliou variáveis radiográficas associadas à progressão da SIFA concluiu que há evidência moderada de que o aumento do ângulo alfa está associado à progressão da doença e que marcadores adicionais não influenciariam. ${ }^{21}$ Neste trabalho encontramos valores altos de ângulo alfa em ambos os quadris, sem diferença significante ao comparar os membros, o que sugere que outros fatores além da alteração femoral influenciam o desenvolvimento do sintoma. Observou-se também que a diferença no índice de retroversão entre os membros foi clínica (26\% maior no membro sintomático) e estatisticamente significante e, certamente, fator determinante para o desenvolvimento de sintoma.

A inibição neuromuscular do quadril é comum em pacientes com SIFA. Os estabilizadores profundos podem ser mais afetados devido sua proximidade com o tecido lesionado, o que sobrecarrega a articulação e todo membro inferior. ${ }^{22}$ Estudo que avaliou força muscular isométrica de 22 pacientes com SIFA comparado a 22 controles correspondentes observou diferença significante na força muscular dos grupos adutores, abdutores, flexores e rotadores externos de quadril no grupo com sintoma. ${ }^{23}$ Outros pesquisadores investigaram a força isométrica e isocinética de indivíduos com SIFA, comparados a controles. Estabeleceu-se a razão entre os grupos agonistas e antagonistas em cada grupo, concluindo que indivíduos com SIFA apresentam $20 \%$ de fraqueza isométrica dos abdutores de quadril e também desequilíbrio na razão entre rotadores laterais e mediais de quadril, quando comparados a outro grupo. ${ }^{4}$ Observamos redução da força muscular nos grupos adutores de quadril e flexores de joelho ao comparar membros, porém clinicamente a diferença foi pequena.

Kockara et al. ${ }^{24}$ concluíram que valores de IMC $\geq 25 / \mathrm{m}^{2}$ podem ser preditores de desfecho clínico desfavorável após tratamento cirúrgico para SIFA. Em nosso estudo o aumento de IMC teve correlação positiva com a perda de RM dos quadris, o que pode indicar uma maior gravidade da SIFA em pacientes com sobrepeso. Embora esta alteração encontrada também possa resultar da dificuldade de avaliação da rotação medial do quadril em virtude do acúmulo de gordura/massa muscular inguinal e abdominal nestes pacientes.

O longo tempo de espera até o diagnóstico foi observado em nossa casuística e de encontro ao estudo de Kahlenberg et al., ${ }^{25}$ que após avaliar 78 pacientes com diagnóstico de SIFA e aplicar questionário padronizado, observou que a duração média entre início dos sintomas e diagnóstico foi de 32 meses. Este achado demonstra a importância de uma avaliação apropriada e diagnóstico oportuno, pois na tentativa de aliviar a dor muitos pacientes podem ser expostos a tratamentos medicamentosos de longo prazo e intervenções incorretas, gerando risco para a saúde e progressão da lesão.
Algumas limitações deste estudo precisam ser consideradas, tais como, a casuística pequena comparada aos trabalhos de grandes centros, avaliação apenas da incidência radiográfica AP de bacia, o que pode não identificar alguns casos de deformidade do tipo came somente visíveis nas incidências em perfil, não estratificação do nível de atividade física em pacientes avaliados ou avaliação dinâmica de força muscular. A principal contribuição deste estudo foi identificar possíveis fatores relacionados à presença de dor e limitação de rotação medial de quadril em pacientes com SIFA. Outros focos sobre avaliação podem criar novas oportunidades de tratamento e acompanhamento desses pacientes.

\section{Conclusão}

Ao comparar características clínicas e radiográficas, observamos redução de rotação medial e aumento do índice de retroversão acetabular no quadril sintomático, bem como associação entre o longo tempo de sintoma e IMC elevado com perda de rotação medial dos quadris.

\section{Conflito de Interesses}

Os autores declaram não haver conflito de interesses.

\section{Agradecimentos}

Agradecemos a colaboração do Dr. Guilherme Guadagnini Falótico pela realização das mensurações radiográficas do artigo.

\section{Referências}

1 Griffin DR, Dickenson EJ, O'Donnell J, et al. The Warwick Agreement on femoroacetabular impingement syndrome (FAI syndrome): an international consensus statement. Br J Sports Med 2016;50(19):1169-1176

2 Ganz R, Parvizi J, Beck M, Leunig M, Nötzli H, Siebenrock KA. Femoroacetabular impingement: a cause for osteoarthritis of the hip. Clin Orthop Relat Res 2003;(417):112-120

3 Diamond LE, Dobson FL, Bennell KL, Wrigley TV, Hodges PW, Hinman RS. Physical impairments and activity limitations in people with femoroacetabular impingement: a systematic review. Br J Sports Med 2015;49(04):230-242

4 Diamond LE, Wrigley TV, Hinman RS, et al. Isometric and isokinetic hip strength and agonist/antagonist ratios in symptomatic femoroacetabular impingement. J Sci Med Sport 2016;19(09): 696-701

5 Malta M, Cardoso LO, Bastos FI, Magnanini MM, Silva CM. Iniciativa STROBE: subsídios para a comunicação de estudos observacionais. Rev Saude Publica 2010;44(03):559-565

6 Pacheco-Carrillo A, Medina-Porqueres I. Physical examination tests for the diagnosis of femoroacetabular impingement. A systematic review. Phys Ther Sport 2016;21:87-93

7 Gradoz MC, Bauer LE, Grindstaff TL, Bagwell JJ. Reliability of Hip Rotation Range of Motion in Supine and Seated Positions. J Sport Rehabil 2018;27(4):1-4. Doi: 10.1123/jsr.2017-0243

8 Sisto SA, Dyson-Hudson T. Dynamometry testing in spinal cord injury. J Rehabil Res Dev 2007;44(01):123-136

9 Bohannon RW, Kindig J, Sabo G, Duni AE, Cram P. Isometric knee extension force measured using a handheld dynamometer with and without belt-stabilization. Physiother Theory Pract 2012;28 (07):562-568

10 Magalhães E, Fukuda TY, Sacramento SN, Forgas A, Cohen M, Abdalla RJ. A comparison of hip strength between sedentary 
females with and without patellofemoral pain syndrome. J Orthop Sports Phys Ther 2010;40(10):641-647

11 Robinson RL, Nee RJ. Analysis of hip strength in females seeking physical therapy treatment for unilateral patellofemoral pain syndrome. J Orthop Sports Phys Ther 2007;37(05):232-238

12 Mohtadi NG, Griffin DR, Pedersen ME, et al; Multicenter Arthroscopy of the Hip Outcomes Research Network. The Development and validation of a self-administered quality-of-life outcome measure for young, active patients with symptomatic hip disease: the International Hip Outcome Tool (iHOT-33). Arthroscopy 2012; 28(05):595-605, quiz 606-10.e1

13 Polesello GC, Godoy GF, Trindade CA, Queiroz MC, Honda E, Ono NK. Tradução e adaptação transcultural do instrumento de avaliação do quadril iHOT. Acta Ortop Bras 2012;20(02):88-92

14 Tannast M, Siebenrock KA, Anderson SE. Femoroacetabular impingement: radiographic diagnosis-what the radiologist should know. AJR Am J Roentgenol 2007;188(06):1540-1552

15 Gosvig KK, Jacobsen S, Palm H, Sonne-Holm S, Magnusson E. A new radiological index for assessing asphericity of the femoral head in cam impingement. J Bone Joint Surg Br 2007;89(10): 1309-1316

16 Diaz-Ledezma C, Novack T, Marin-Peña O, Parvizi J. The relevance of the radiological signs of acetabular retroversion among patients with femoroacetabular impingement. Bone Joint J 2013;95-B(07): 893-899

17 Garcia AS, Gobetti M, Tatei AY, Falótico GG, Arliane GG, Puertas EB. Prevalência de sinais radiográficos de impacto femoroacetabular em indivíduos assintomáticos e não atletas. Rev Bras Ortop 2019; 54(01):60-63
18 Tak I, Engelaar L, Gouttebarge V, et al. Is lower hip range of motion a risk factor for groin pain in athletes? A systematic review with clinical applications. Br J Sports Med 2017;51(22):1611-1621. Doi: 10.1136/bjsports-2016-096619

19 Audenaert EA, Peeters I, Vigneron L, Baelde N, Pattyn C. Hip morphological characteristics and range of internal rotation in femoroacetabular impingement. Am J Sports Med 2012;40(06): 1329-1336

20 Rhee C, Le Francois T, Byrd JWT, Glazebrook M, Wong I. Radiographic Diagnosis of Pincer-Type Femoroacetabular Impingement: A Systematic Review. Orthop J Sports Med 2017;5(05): 2325967117708307

21 Wright AA, Naze GS, Kavchak AE, Paul D, Kenison B, Hegedus EJ. Radiological variables associated with progression of femoroacetabular impingement of the hip: a systematic review. J Sci Med Sport 2015;18(02):122-127

22 Retchford TH, Crossley KM, Grimaldi A, Kemp JL, Cowan SM. Can local muscles augment stability in the hip? A narrative literature review. J Musculoskelet Neuronal Interact 2013;13(01):1-12

23 Casartelli NC, Maffiuletti NA, Item-Glatthorn JF, et al. Hip muscle weakness in patients with symptomatic femoroacetabular impingement. Osteoarthritis Cartilage 2011;19(07):816-821

24 Kockara N, Sofu H, Issin A, Çamurcu Y, Bursali A. Predictors of the clinical outcome and survival without degenerative arthritis after surgical treatment of femoroacetabular impingement. J Orthop Sci 2018;23(01):117-121

25 Kahlenberg CA, Han B, Patel RM, Deshmane PP, Terry MA. Time and Cost of Diagnosis for Symptomatic Femoroacetabular Impingement. Orthop J Sports Med 2014;2(03):2325967114523916 